\title{
Artificial Intelligence (AI) Based on Eccentricities
}

\author{
Santiago Esteva*, Albert Figueras and Josep Llluís De la Rosa
}

VICOROB Institute, University of Girona, Spain

\begin{abstract}
Into the highest level of invention or discovery, we find that many of humanity's advances have been achieved by eccentricities of its author, where he has tried to imagine or create something different from what can be generated by deduction, imitation or reasoning, which they are already elaborate forms of intelligence. In this work is proposed to join one last layer after the abstracting information of the data of the process, and the first level of the intelligence, like NN, any classification methods, reasoning etc., a new layer of the analysis of this information to search some matching with the eccentricities proposed, this can produce one result that can be seen as a great level of intelligence if this is according to the hypothesis. The premises and viability are also analyzed to be viable the proposed hypothesis.
\end{abstract}

Keywords: Artificial intelligence $•$ Neural network $•$ Eccentricities

\section{Introduction}

The artificial intelligence have been developed to be used in many new areas, started in a automatic control then in a robot navigation and recently in a cars and in a facial recognition etc. Also the algorithms have been changed, augmenting the quantity of the neurons in a NN, and mixing different techniques, clustering, genetic, stochastic algorithms, reasoning, and learning possibilities [1]. All these give huge potential at new applications. In this work, we focus to some new paradigms, as follows:

We understand as environmental intelligence, the knowledge that the human being has in a known environment of interaction between machines, tools, materials, space, etc., with which the operator knows exactly how to act, for example, after a problem arisen suddenly and new, there exist methods for isolation and fault detection, but not for the automatic resolution of them. We know that it is possible to improve the automatic resolution of the problems that have arisen, increasing the sensing of the environment, so that artificial intelligence can contribute more knowledge to the solution of the problem [2].

Superintelligence as an agent element [3] that encapsulates information and methodology, with capacity for artificial intelligence among the cooperating elements, which can overcome the brilliance of a trained human, leads to the development of skills and abilities of an inventor or artist. It is also being discussed if a robot or artificial intelligence should be entitled to patents or copyrights, for this the machine must be able to generate new tools or artistic or scientific creations, which is where we want to get to this work.

The machine by artificial intelligence aims to discover or invent something that the programmer himself cannot accurately observe and predict. With this, the process will provide the data that will be used to adjust the algorithm parameters and obtain the appropriate results, therefore, the programmer by introspection can determine the formulae or the configuration method, it seems that it is not obvious, when there are large numbers of weights adapted by the methodology and parts of the enhancer algorithm, plus the interaction between the parts and theirscheme.
How to invent or create something new is possible, in this paper we present an upper layer of artificial intelligence as shown in Figure 1, where the result of a neural network that already makes its decisions, for example, to determine whether it is a dog or a cat the image, and through for more information and this result, for example, indicate the character of that animal. For this, it is necessary to start from a hypothesis and provide an algorithm that represents it, and adjust the parameters through learning so that the new experiments obtain favorable results, as is the case of the new scientific theories that must at least be validated by the experimentation.

\section{Eccentricities}

By climbing to the highest level of invention or discovery, we find that many of humanity's advances have been achieved by eccentricities of its author, where he has tried to imagine or create something different from what can be generated by deduction or reasoning, which they are already forms of intelligence. Starting from the basic forms of learning, by discovery, imitation and deduction, as well as the stages of learning of the human being in its first states, and then together with the accumulated knowledge, it allows the human being to predict new situations from the previous knowledge, and reason to deduce behaviors using new measuring devices, raise and find new knowledge from the analogy of what happened before.

At the end of extracting knowledge from the previous information and the memory of cases, the capabilities of the human being are limited, that is why it is necessary to resort to the imagination, to invent the impossible with the acquired information and the existing relationships. A process that can lead to a significant loss of time and resources, since surely they will be inadequate, it must be verified that the hypothesis cannot be solved based on accumulated knowledge, a situation that would have already been determined if it were the result of a logical inference. You should also check that the hypothesis is not negative for the use of existingknowledge.

There exist $x$ to verifies $P(x) ;(x) P(x)$ And also to verify $(x) \neg P(x)$

If any of these cases is effective, it will be resolved as an invalid or nonnovel hypothesis. In the rest, it will be necessary to see if any of the result is

"Corresponding author: Santi Esteva, VICOROB Institute, University of Girona, Spain, Tel: +34 626411206; E-mail: santiesteva@gmail.com

Rec date: March 17, 2020; Acc date: April 16, 2020; Pub date: April 23, 2020

Copyright: ๑ 2020 Esteva S, et al. This is an open-access article distributed under the terms of the Creative Commons Attribution License, which permits unrestricted use, distribution, and reproduction in any medium, provided the original author and source are credited. 
valid and consistent, which will have obtained new information or knowledge and it can be considered as somethingintelligent.

Example: suppose a chemist who proposes a new mixture of products and processes that can produce a synthetic material. First, it is to assess whether the proposed method is positive with existing knowledge, which has not yet been experienced. Then verify that with the existing knowledge it is possible to reach the non-hypothesis. The next step will be to try, from the existing knowledge, to suppose a new form for the realization of the hypothesis, which will surely have different possibilities, some impossible, some possible and one of them optimal, that would be the desirable result, and with that it would have expanded prior knowledge and could be considered as a new and intelligent step.

Many advances in science arise as a result of new and better measuring devices, so that existing knowledge can be applied to new measurements, for example, as more powerful microscopes are inferred in the behavior and relationship between cells, or chemical elements or atomic particles for example. In some of these cases, before the elements could be observed, there were already theories about their possible existence and their relationships, and it was as a result of extravagant hypotheses, but which they fulfilled without being incompatible with the existing and partially incomplete rules of knowledge.

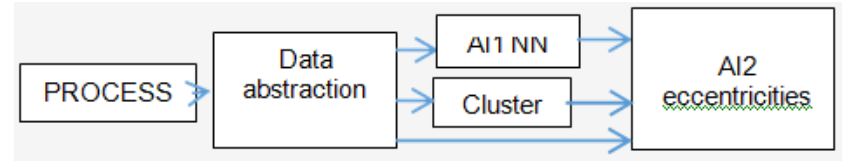

Figure 1: Processing schema.

The application of eccentricities in artificial intelligence systems, needs to incorporate multiple artificial intelligence methods, such as neural networks, expert systems, classification algorithms, statistics, etc., that works with pure data and abstractions of them configuring multiple layers of information and incorporating all this at different levels, new relationships between the various elements can be generated to obtain a new result.

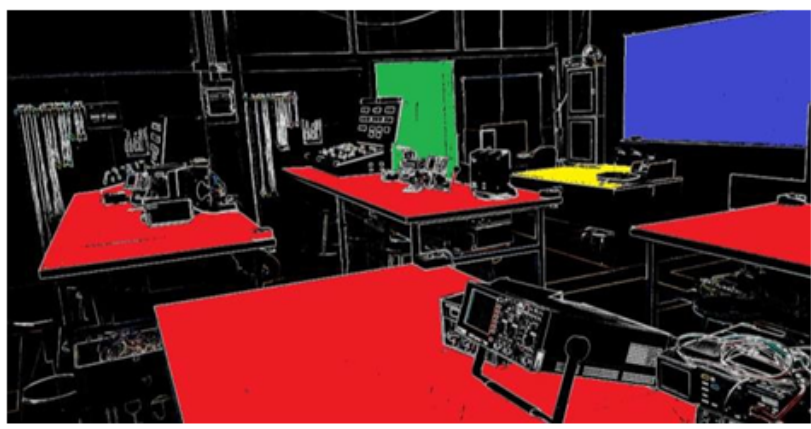

Figure 2: Laboratory image.

For example, in a system of visual recognition, from an image, the extraction of vertices, masses, position, distances, etc., and the subsequent recognition of forms by neural networks and the characteristic elements of a laboratory as in Figure 2, by adding a layer to determine by position and distribution of these elements, information of the use of the laboratory as a hypothesis. In case of a good functioning, it would be considered as an intelligent system if, from the image, the name of the laboratory in question of the analyzed University was informed, without having previous images of it. The hypothesis could be the design criteria of the different types of laboratory, by equipment and desired functionality. In this case, the incompatibility of the hypothesis with the existing information is done by analyzing that the design criteria with the existing cases do not allow a complete segmentation of the varioussituations.

Starting with the result of applying filters in the image looking for contours and shapes, then applying neural network structures to determine sets of objects to allow define laboratory characteristics. And using the classification of data in a group to determine the number of elements that makes up each group of objects and also using the width of the free spaces. Then, all these results will be treated by another layer of neural networks to classify with these inputs the different types of laboratories, this is possible using Kohonen's self- organized neural networks. This experiment would allow unsupervised learning to reinforce the classification for each of the images presented differently.

Applying an eccentricity and assigning a result to the laboratory as an assignment of weights to certain characteristics of the laboratory, such as distribution, free space, hallways, brightness, color, etc., by learning how to adjust the weights of the different entries, using some existing cases to give the efficiency of the laboratory with a percentage of students who pass the subject. Result that is assigned to an image of the laboratory analyzed with its students, which would cause a human to try to obtain said result as an analysis of the faces of the students to determine by the face the intellectual and labor capacity of the students. Whose case is independent of this, if the number of students is significant, and depends solely on the type of subject, ergonomics, hours of work, etc. Even on another level, it can be proposed to determine which students pass the subject, with a hypothesis of distance to the teacher's table or the board, distance to the nearest student who forms work groups, or distance to the exit door, and elements used in the workplace, all with standardized data to the analyzed laboratory, in order to enter a trained and replicated neural network for each student. If these results were correct, it would be interpreted as an intelligent system by being able to determine from a photograph of any laboratory, the students who will overcome thesubject.

Specific example, if we want to classify prime numbers, based on a hypothesis to facilitate classification in the general numerical space of those who are prime to group them into areas with common characteristics and introduce the existence of the remainder as inputs to the neural network of the number divided by 2, 3, 5 and 7 and the product, and we proceed to the training of the network and it gives $70 \%$ correct, it will be possible to ensure that the hypothesis is suitable for this case, and more if the entries are extended to other dividends, the result hardly improves.

\section{Control of Al}

The objective to be determined by the higher intelligence layer can be diverse and variable, where one objective or another can be sought depending on its difficulty. For example, a humanoid robot tries to interact with a human and manages to receive stimuli of interaction through natural language processing. If there are too many contact stimuli in the conversation, hugs or hand crashes, the search for this type.

At the same time, said higher intelligence layer generates a suboptimal solution result, and in some cases optimal. If another similar upper layer is added, the experimentation would start from a different random point and again allow some cases to be enhanced to obtain the optimal result. For example, if this robot wanted to be relevant in society, it could attempt communication as a humorist, as a parody of celebrities, as a political leader or finally as a dictator, in which case it would attract the attention of much of society and television media.

The theory that the robot responds exactly to what its programmer has designed and assumed, in this case it is very diffuse, first because the objective can be a composition of objectives to be automatically weighted, and also the use of several layers of intelligence in which the various information elements will be weighted, and it will establish a solution around a local maximum and will make unpredictable the behavior that the robot will take. Unpredictable results due to a priori ignorance of all possible local maximums. 


\section{Application}

The application given to our autonomous robots that cross urbanizations is the collection of artificial public lighting data as in Figure 3 [4]. The use of neural networks in the control of navigation has long been used [5], we also apply artificial intelligence [6] to the navigation, where you must recognize objects and elements on the trajectory, some to organize the route overcoming obstacles, and others in those who pay more attention, they intuit that they can be insurmountable obstacles, all these to take the measures more correctly after recognizing the lighting elements and in very different lighting conditions. A higher degree of intelligence will take into account the obstacles that arise and decide whether they should be overcome or avoided, which depends not only on size, but also on shape, stability, material, etc., as in Figure 4. As with human beings, we instinctively step on tiles that are not firm or avoid them without thinking or deciding a priori their degree of danger.

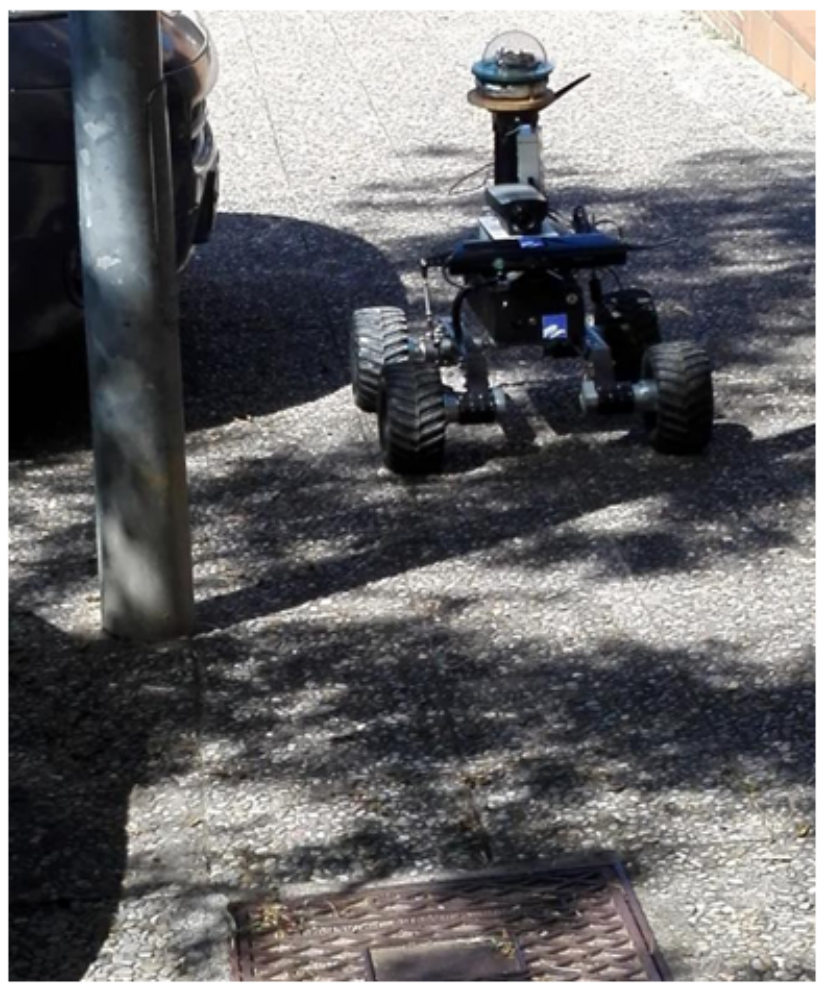

Figure 3: Autonomous robot navigation.

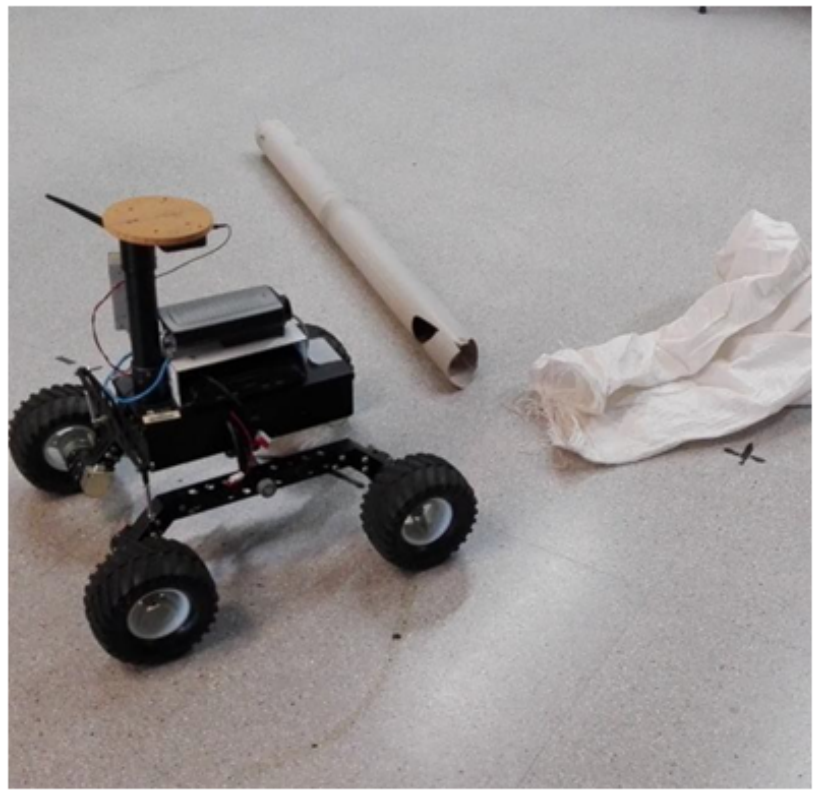

Figure 4: Danger recognizion.

\section{Conclusion}

The objective of this work is to analyze existing artificial intelligence systems to be used as inventors or idealize them to expand imaginary ideas. Some experiments present how to implement and analyze its feasibility and results, and try to generate a discussion and a topic to be treated as a new area of artificial intelligence.

\section{References}

1. Domingos, Pedro. "The master algorithm: How the quest for the ultimate learning machine will remake our world." Basic Books, 2015.

2. Robinson, David Charles, David Adrian Sanders, and Ebrahim Mazharsolook. "Ambient intelligence for optimal manufacturing and energy efficiency." Assembly Automation (2015).

3. DE, Christian G Quinterom Josep LI, and Josep Vehi La Rosa. "Physical Intelligent Agents' Capabilities Management for Sure Commitments in." Recent Adv Artificial Intelligence Res Dev 113 (2004): 251.

4. Figueras Albert, Josep Lluís De La Rosa, Santiago Esteva, and Xavier Cufí. "Robot team in the improvement of the neighborhood." In 2018 IEEE International Smart Cities Conference (ISC2), pp (2018): 1-6.

5. Esteva, Santiago, A Oller, C Pous, and J Armengol. "Comparing Neural Networks and Fuzzy Control Techniques." (1996).

6. Figueras, A, Santiago Esteva, X Cufí, and J LL De La Rosa. "Applying Al to the motion control in robots. A sliding situation." IFAC-PapersOnLine 52 (2019): 393-396.

How to cite this article: Esteva S, Figueras A, and De la Rosa JL. "Artificial Intelligence (Al) Based on Eccentricities". J Comput Sci Syst Biol 13 (2020) doi: $10.37421 / \mathrm{jcsb} .2020 .13 .309$ 